
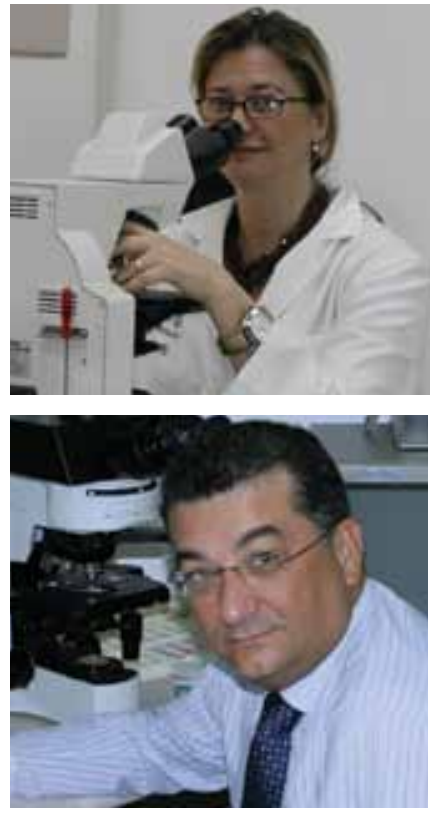

Palabras clave

Educación médica, patología, enseñanza multimedia, blended learning

\section{Key Words}

Medical education, pathology, multimedia pedagogy, blended learning

Recibido el 21-May-11 Aprobado el 28-May-11

\title{
Integración teórico-práctica en un contexto de "blended-learning" para mejorar la transferencia de conocimiento. La experiencia de Patología
}

\author{
Theoretical and practical integration in a blended-learning context as a tool to \\ enhance knowledge transfer. Our experience in the area of Pathology
}

\section{María José Fernández* y Félix Contreras ${ }^{\star *}$}

Resumen: Este trabajo recoge la experiencia de la reconsideración del proceso de enseñanzaaprendizaje de la Patología en la Pontificia Universidad Católica Madre y Maestra. Basándonos en fuentes pedagógicas, en fuentes profesionales y en nuestra propia experiencia, indagamos sobre el uso de nuevos métodos. Desde una perspectiva cognitiva, estudiamos el uso de estrategias didácticas con énfasis en la elaboración de materiales didácticos multimedia. Se trata de alcanzar, en el área de Patología, la mejor transferencia del conocimiento desde los cursos básicos a los cursos clínicos, así como la mejor transferencia del conocimiento teórico a la práctica y viceversa para alcanzar aprendizaje.

Abstract: This article presents the experience of implementing new teaching strategies in the Pathology courses offered at Pontificia Universidad Católica Madre y Maestra. The aim of introducing these changes is to achieve, in the area of Pathology studies, the best knowledge transfer possible all the way from basic courses to clinic courses, as well as the best transfer possible from theoretical knowledge to practice and vice versa. From a cognitive perspective and based on pedagogical and professional sources, as well as on the authors' experience a new teaching methodology was approached. The authors studied the use of teaching strategies with an emphasis on the elaboration of multimedia didactic materials and their influence on students' learning.

\section{Introducción}

Con la meta de mejorar la calidad del aprendizaje de la Medicina, hemos diseñado y aplicado un nuevo sistema de enseñanza de la Patología en la Pontificia Universidad Católica Madre y Maestra que incluye una reconsideración de las estrategias didácticas ${ }^{1}$ a emplear en cada momento de su enseñanza y una organización integrada con las demás materias y entre los distintos profesores.

Basándonos tanto en las fuentes pedagógicas como en las fuentes profesionales y, en nuestra propia experiencia, hemos indagado sobre el uso de nuevos métodos didácticos incluyendo el uso de plataformas digitales. En este contexto, y desde una perspectiva cognitiva, estudiamos el diseño de programas y de estrategias didácticas con especial énfasis en la elaboración de materiales didácticos, incluyendo multimedia.

Nuestro objetivo ha sido sistematizar de manera integral la materia Patología, en las intenciones y los contenidos, las estrategias de enseñanza-aprendizaje y los recursos para lograr un mejor aprendizaje de la Medicina. La experiencia permite proponer esta didáctica de la Patología como un ejemplo de transferencia eficaz, según las teorías cognitivas.

\footnotetext{
"Doctora en Medicina por la Universidad Central del Este, Rep. Dominicana. Magíster en Pedagogía Universitaria por la Pontificia Universidad Católica Madre y Maestra. Profesora de Patología en esa misma Universidad. Para contactar a la autora: majfr@hotmail.com
}

\begin{abstract}
"Doctor en Medicina por la Universidad Autónoma de Madrid. Magíster en Pedagogía Universitaria por la Pontificia Universidad Católica Madre y Maestra, Campus de Santiago. Decano de la Facultad de Ciencias de la Salud y profesor de Patología
\end{abstract} de esa misma universidad. Para contactar al autor: f.contrerasmejuto@gmail.com

${ }^{1}$ Cecilia Bixio (2005) define las estrategias didácticas como "el conjunto de acciones que realiza el docente con clara y explícita intencionalidad pedagógica".

Encuentre el texto en "Estrategias de enseñanza-aprendizaje" http://www.pucmm.edu.do/STI/campus/CDP/ComunicaciónPublicaciones/Paginas/CuadernodePedagogiaUniversitaria.aspx

Cuaderno de Pedagogía Universitaria Año 8/ N. 15 /enero - junio 2011 / Santiago, Rep. Dom.: PUCMM / pp.21-26 
Este artículo se estructura en cuatro secciones: 1) Fundamentos conceptuales: la concepción disciplinar y didáctica de la Patología, así como la justificación de su enseñanza en Medicina. 2) Las intenciones y los contenidos: revisamos los objetivos mínimos de aprendizaje de la Patología, los contenidos teóricos y prácticos, y su distribución dentro del pénsum. 3) Las estrategias de enseñanza-aprendizaje: propusimos nuevos abordajes pedagógicos para alcanzar los objetivos. Diseñamos y elaboramos los materiales didácticos a utilizar incluyendo el uso de plataformas en línea. 4) Los recursos: adaptamos un espacio modelo adecuado para la implementación de la nueva propuesta didáctica identificando y analizando las características imprescindibles para el desarrollo de técnicas docentes adaptadas a las teorías cognitivas que pretendemos desarrollar.

\section{Fundamentos conceptuales La concepción disciplinar y didáctica de la Patología}

La Patología, como se conoce en los países anglosajones y, por influencia de estos, en la mayor parte de lbero América, o "Anatomía Patológica", término más tradicional aunque restrictivo, como se conoce en España, Francia y otros países europeos, es una ciencia morfológica, básica y al mismo tiempo aplicada, que forma parte de las Ciencias de la Salud. La Patología estudia los procesos de la enfermedad desde un punto de vista morfofuncional y molecular. Es, por lo tanto, la rama de la Medicina que nos permite entender las causas y los mecanismos íntimos de todos los procesos de enfermedad. Sin conocer y comprender bien esos mecanismos y procesos resulta imposible entender bien la clínica o la terapéutica de las diferentes enfermedades. Como ciencia morfológica básica, es una materia básica de la carrera de Medicina. En este sentido es también un campo de investigación básica de procesos de enfermedad celulares, subcelulares y moleculares.

Por otro lado, desde la década de los 50, la Patología se ha convertido también en una especialidad médica en el ámbito asistencial puesto que el estudio morfológico y molecular de especímenes obtenidos de los pacientes es esencial e imprescindible para establecer un diagnóstico correcto o para confirmar una sospecha diagnóstica, para establecer el pronóstico, para colaborar en la decisión terapéutica, y para valorar la respuesta a esta (Rosai, 1997). En este sentido la Patología representa también un control de calidad de la medicina clínica y un campo de investigación más aplicado (clínicopatológico) con estrecha relación incluso con el desarrollo o evaluación de nuevas terapéuticas.

Es por esta "doble cara" de la Patología, básica y asistencial, que está presente en las facultades de medicina como un departamento o área básica, con líneas de investigación básica, y por otro lado está presente en hospitales y clínicas como un departamento diagnóstico involucrado directamente en la atención del paciente, con líneas de investigación aplicada.

Dada esa doble faceta de la Patología que hemos mencionado, es preciso contar con una actitud o una habilidad peculiar del docente. Hay que demostrar continuamente al estudiante que el profesor de Patología no es un simple conocedor teórico, sino un conocedor y un hacedor. Es decir, que los conocimientos que imparte y exige, no son abstractos y estáticos y que de hecho tienen una aplicación práctica médica concreta. Al explicar la Patología, hay que hacer ver continuamente que esos conocimientos son la base del método anatomoclínico que es la herramienta de trabajo de la medicina actual. El profesor de Patología no debe perderse en el detalle de la morfología de las lesiones sino, por el contrario, debe hacer énfasis en su significado y en su utilidad asistencial (Contreras, 1996).

Justificación de la enseñanza de la Patología en la carrera de medicina Una vez definida la Patología y su campo de acción podemos tener una idea del contexto epistemológico y del contexto de significación de esta materia. En definitiva, ¿qué aporta la Patología al estudiante de Medicina y a su perfil profesional de médico?

Como ya hemos mencionado, la Patología es una materia esencial para comprender los procesos básicos de la enfermedad y, por lo tanto, imprescindible para entender, sustentar y elaborar todo el aprendizaje posterior de las diferentes ramas clínicas de la Medicina. Sin un conocimiento profundo de la Patología General el estudiante de Medicina podrá adquirir conocimientos médicos sobre las enfermedades así como destrezas y habilidades diagnósticas o terapéuticas pero nunca llegará a comprender el cómo y el porqué de esas enfermedades que diagnostica mecánicamente. La única diferencia entre él y un curandero será donde aprendieron esos conocimientos y que lo que él aprendió está aceptado por la comunidad médica o científica pero carecerá, al igual que aquel, de explicaciones sobre la naturaleza de los procesos ante los que se enfrenta. Difícilmente podrá elaborar nuevos conocimientos autónomos o aportes científicos porque carece de una base sólida sobre la que apoyarlos. Por otra parte, el estudiante de Medicina que, a través de las clases o seminarios de Patología Especial, comprende el papel que la Patología tiene actualmente en el diagnóstico, pronóstico y tratamiento del paciente, se convertirá en un Médico que sabrá utilizar esta "herramienta" correctamente en beneficio de los pacientes. No se trata de que el Médico sea un Patólogo, sino que sepa qué puede pedirle al Patólogo, cuándo debe pedírselo y cómo debe interpretar la información que este le suministre.

Ausubel en su conocido libro Psicología educativa. Un punto de vista cognoscitivo y a lo largo de todas sus publicaciones, siempre ha utilizado como ejemplo sobresaliente de diferenciación progresiva el libro de texto Patología (Texbook of Pathology) de Boyd (1961). En él, Boyd se desliga de los tratados de Patología que suelen constar de más de 200 capítulos, cada uno dedicado a describir en serie las clases principales de procesos patológicos que ocurren dentro de un organismo o sistema de órganos en particular. Boyd, en contraste con estos, reserva la consideración en serie de la patología de los diversos órganos para la segunda parte del libro y consagra toda la primera mitad a asuntos de organización e integración generales como son las diferentes categorías de procesos patológicos (inflamación, alergia, degeneración, neoplasia), y a las causas y características principales de estos; las diversas clases de agentes etiológicos de las enfermedades; los tipos de resistencia humoral y tisular a la enfermedad; la interacción de los factores genéticos y ambientales en el desarrollo de los procesos patológicos; y las relaciones generales que hay entre las lesiones patológicas y los síntomas clínicos. abarquen.

Integración teórico-práctica en un contexto de "blended-learning" para mejorar la transferencia de conocimiento, María José Fernández y Félix Contreras Cuaderno de Pedagogía Universitaria Año 8/ N. 15 / enero - junio 2011/ Santiago, Rep. Dom.: PUCMM / pp.21-26 
Cabría preguntarse, como Du Boulay (1997): ¿por qué enseñar Patología?, ¿cómo enseñar la Patología? y ¿cuándo enseñar la Patología? Quizá, como hemos visto ya, la respuesta a la primera pregunta sea la más fácil. La Patología General proporciona la base para la comprensión de la Medicina Clínica.

\section{Las intenciones y los contenidos}

La Patología debe de dar un significado pragmático, concretamente médico, a los conocimientos que ha adquirido el estudiante en las unidades precedentes y por otra parte que adquiera una base sólida del substrato morfológico de la enfermedad. El programa en su contenido y en su desarrollo está orientado a que el estudiante asimile el concepto del método anatomoclínico como herramienta imprescindible en el ejercicio futuro de su profesión y que aprenda a utilizarlo desde esta etapa inicial de su carrera universitaria.

Una parte importante de un programa es el de las lecciones teóricas a impartir en forma de lecciones magistrales, pero en Patología el aprendizaje no será el adecuado si no se acompaña de suficiente número de horas de enseñaza práctica. El aprendizaje es más significativo si teoría y práctica están integradas en tiempo y en espacio. Esta integración motiva en gran medida a los estudiantes, facilita y estimula el uso de multimedia, permite una aplicación directa y efectiva de materiales en línea, y aumenta notablemente la interacción y la participación.

De acuerdo con esta premisa, el programa que presentamos integra de manera indivisible los componentes teórico y práctico, y estructura la materia en tres partes:

- Patología General I

- Patología General II

- Patología Especial (se imparte integrada con las diferentes asignaturas clínicas)

La Patología General I (MED-371) pretende conseguir que el estudiante asimile el concepto de Patología Celular y de acuerdo con él, aprenda la morfopatología básica de la enfermedad. La Patología General II (MED-375) se divide en tres bloques: Neoplasia, Patología Infecciosa y Patología Ambiental. Se pretende conseguir que el estudiante asimile el concepto de Neoplasia desde el punto de vista de la Patología Celular y Molecular. El bloque de Patología infecciosa analiza la forma de reaccionar frente a los distintos tipos de agentes infecciosos. Por último, en el bloque de Patología Ambiental se revisan algunas causas y mecanismos de toxicidad por exposiciones a agentes ambientales y profesionales. La enseñanza de la Patología Especial se integra en los cursos médico-quirúrgicos de los diferentes aparatos y sistemas.

\section{Las estrategias de enseñanza-aprendizaje}

Una de las preocupaciones de la Educación Médica es descubrir modos eficaces de que los estudiantes adquieran la gran cantidad de conocimientos de base necesarios para la práctica de la Medicina actual de un modo tal que puedan retenerlos por largo tiempo en condiciones funcionales, viables, necesarias para su aplicación con éxito a los problemas de la práctica clínica (Ausubel, 1980).
En la educación actual el centro del proceso enseñanza-aprendizaje deja de ser el profesor y pasa a ser el estudiante. Los docentes ceden el escenario, el protagonismo, la palabra, a los estudiantes, de modo que la educación centrada en la enseñanza pasa a ser centrada en el aprendizaje. Desde ese punto de vista, las estrategias didácticas que se están consensuando en el espacio común europeo (propuestas de Bolonia) y que se materializan en el proyecto "tuning" están encaminadas precisamente hacia un aprendizaje centrado en el estudiante que tenga en cuenta tanto los elementos presenciales como la autoformación no presencial.

Al elaborar las estrategias didácticas de nuestra materia, la Patología, pretendemos abarcar tanto el componente presencial como el no presencial del aprendizaje. Los modos de enseñar así como los materiales de enseñanza están encaminados a facilitar ambos componentes del proceso de una manera integrada y homogénea. La enseñanza, por tanto, tiene características mixtas haciendo un amplio uso del multimedia y de las TIC.

El término blended learning (B-Learning) corresponde a lo que solemos llamar modelo mixto, desde hace ya años. Lingüísticamente hablando, la definición del término "blended" será mezclado o combinado. Por tanto, el blended learning no es ningún invento nuevo asociado a la era del e-learning, sino que tiene tanta antigüedad como la educación a distancia e incluso como el diseño de "tareas para casa" del aprendizaje presencial tradicional. Las fases a distancia de un modelo mixto o blended learning, se desarrollan haciendo uso de diferentes media y de las TIC.

Al hablar de blended learning no pensamos en cursos 100\% presenciales o 100\% a distancia, sino que nos gusta considerar el B-learning como un sistema flexible de formación presencial apoyado en las TIC que permite, según las circunstancias y las características de cada acción formativa, combinar diferentes elementos didácticos: la instrucción clásica (presencial), la realización de actividades, los contactos en tiempo real (presenciales, videoconferencias, chats...) y los contactos diferidos (consultas al profesor, foros de debate, correo electrónico...), etc.

Al tratar de llevar a nuestra práctica docente los principios de las teorías cognitivas del aprendizaje multimedia de Mayer (2009), hemos optado por una visión centrada en el modo de presentar los materiales didácticos con un abordaje centrado en el estudiante. La pregunta que nos hicimos fue: ¿cómo podemos adaptar los multimedia para mejorar en el aprendizaje en la carrera de Medicina? El B-learning como hemos visto previamente es un abordaje que combina el E-learning con enseñanza tradicional dirigida por un profesor donde, por ejemplo, una clase se suplementa con materiales en línea.

El E-Learning con multimedia incrementa tanto el aprendizaje como la enseñanza. Hay evidencias que sugieren que el E-learning es más eficiente porque los estudiantes adquieren conocimientos, habilidades y actitudes más rápidamente que a través de métodos más tradicionales (Ruiz y cols, 2006). Los multimedia proporcionan a los estudiantes la flexibilidad de seleccionar diferentes medios según su estilo de aprendizaje. El B-learning tiene la particularidad de producir un aprendizaje

Integración teórico-práctica en un contexto de "blended-learning" para mejorar la transferencia de conocimiento, María José Fernández y Félix Contreras Cuaderno de Pedagogía Universitaria Año 8/ N. 15 / enero - junio 2011 / Santiago, Rep. Dom.: PUCMM / pp.21-26 


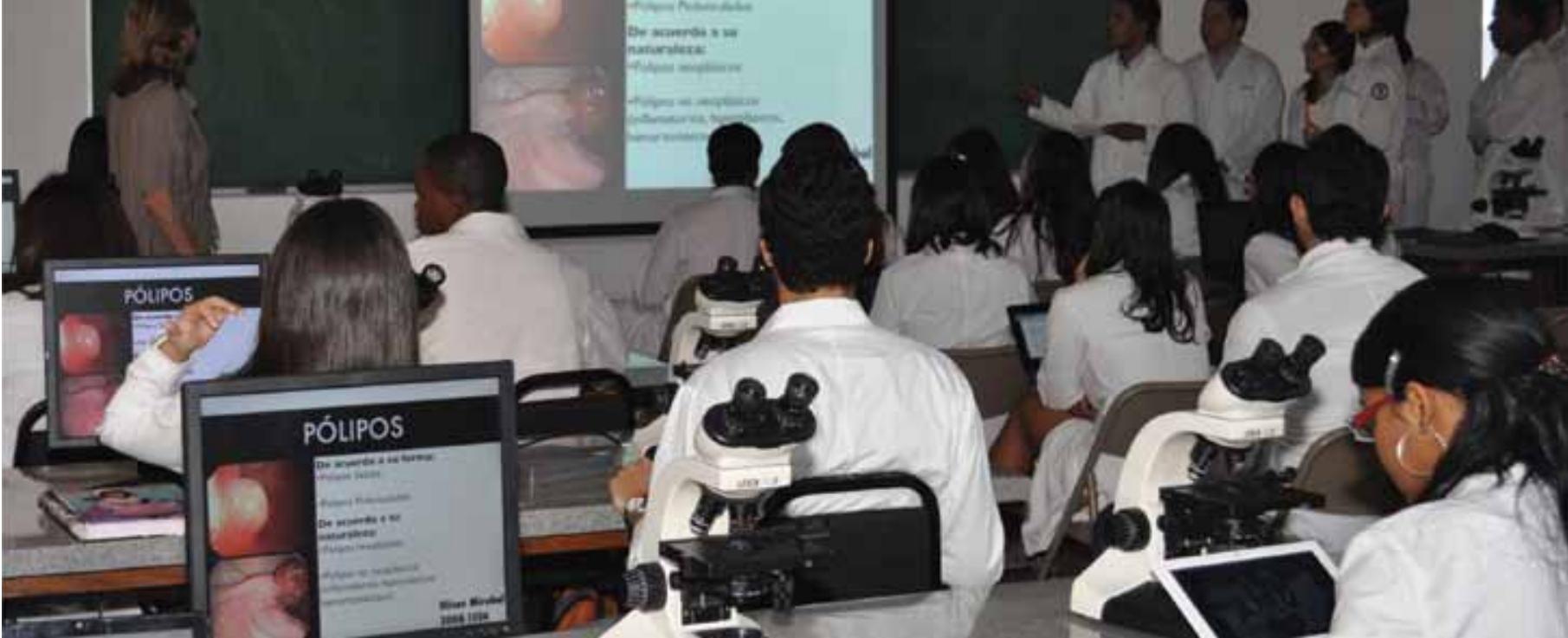

síncrono o asíncrono según la fase presencial o no presencial de este. En nuestra propuesta empleamos la página web de la Unidad Docente de Patología como soporte que puede ser utilizado en ambas fases.

La enseñanza multimedia interactiva es particularmente adecuada para el aprendizaje de la Patología ya que maneja imágenes e información de manera muy eficiente. En varios estudios se ha descrito que los estudiantes prefieren un formato de aprendizaje mixto ("blended learning") que combine la tecnología informática (ordenadores, Internet, etc.) con el abordaje microscópico tradicional (Sharpe y cols. 2006, Bloodgood y Ogilvie, 2006).

En la fase presencial en el aula el profesor adopta un papel de facilitador y en diferentes momentos utiliza diferentes modalidades de enseñanza: clases magistrales, clases interactivas con fuerte uso de imágenes apoyado tanto en la plataforma como en la observación directa del microscopio y resolución de casos problema basados en la Web. El ritmo de las clases presenciales está diseñado y marcado por el profesor, que dispone de tiempo limitado por el horario. Sin embargo, en la fase no presencial, es el estudiante el que decide el momento, el lugar y el ritmo de su propio aprendizaje.

En la fase no presencial, a través de la plataforma, el estudiante dispone de la misma combinación de texto e imagen que el profesor utilizó en la clase presencial y puede utilizarlo a su propio ritmo. Además, dispone de ejemplos que combinan narración y animación (videos y animaciones), colecciones de casos-problema prácticos, archivos de imágenes basadas en la Web y evaluaciones en línea que serán retroalimentadas por el profesor.

La plataforma dispone también de múltiples canales de comunicación con el profesor, como pueden ser el correo electrónico, blogs informativos del profesor, e incluso de otras herramientas web 2.0 del tipo de redes sociales como Facebook o de microblogging como Twitter.

\section{La plataforma de aprendizaje}

La plataforma de aprendizaje está basada en una página Web con la siguiente dirección: http://www.contrerasrobledo.com/docencia. Para la construcción de la página tuvimos muy en cuenta los aspectos didácticos fundamentales que guían nuestra enseñanza, la teoría cog- noscitiva de aprendizaje multimedia y las aplicaciones a la enseñanza de la Patología que abordamos en capítulos previos.

Se entra a través de una página principal y partiendo de ella se accede a múltiples vínculos e hipervínculos. En muchas de las páginas existen vínculos directos con el correo electrónico de los profesores para que la comunicación pueda ser de fácil acceso.

A partir de la página de inicio existen links con:

- Ubicación.

- Patología en la carrera.

- Objetivos mínimos de aprendizaje. Patología I, Patología II y Patología Especial.

En la Página de cada asignatura se dispone de links con:

- Profesor y medios de contacto.

- Filosofía del curso.

- Calendario imprimible.

- Evaluaciones y calificaciones.

- Bibliografía y metodología de estudio con recomendaciones al respecto.

- Programa de la asignatura con la distribución.

En la página de cada tema, el usuario dispone de un menú de navegación y de una barra de imágenes correspondientes a dicho tema, seleccionadas para hacer más atractiva la página y para permitir un vistazo rápido de los cambios morfológicos más relevantes.

Hay links con una página para ver las imágenes y textos de la presentación del profesor en la clase presencial, con videos o animaciones acompañados de narración, con las historias clínicas e imágenes de los casos prácticos que se estudian con el microscopio, con casos prácticos interactivos en otras páginas web, con bibliotecas de imágenes de otros sitios y con una auto evaluación sobre el tema.

Para facilitar la construcción del aprendizaje se han recogido piezas macroscópicas y colecciones de preparaciones histopatológicas para que los estudiantes, mediante su visualización directa y con el microscopio, apliquen en la práctica los conocimientos teóricos adquiridos. De cada caso el estudiante dispone en la plataforma de una historia clínica, de datos complementarios de laboratorio y de imágenes representativas de alta calidad para su repaso fuera del aula. 


\section{Los recursos}

Nuestro interés era transformar el aula de clase, esto surge porque entendemos que es una forma de enriquecer el proceso de aprendizaje, y que posibilita una verdadera mejora en la calidad, mediante la disposición de nuevos medios y materiales, y la diversificación de metodologías en el proceso de enseñanza (Sarsa Garrido, 2008), además nos permite integrar de manera efectiva el nuevo diseño teórico-práctico de las asignaturas Patología General I, II y Especial.

Creemos que la posibilidad de integrar software comercial en el aula, materiales creados por los propios profesores, disponer de conexión a Internet, Ethernet etc. (en sintonía con Reparaz y otros, 2000), son actuaciones que encaminan a una docencia más enriquecedora y llena de actividades significativas como el encuentro más fácil con diversos materiales y recursos, el acceso a la curiosidad y experimentación, el trabajo cooperativo y la expresión libre de los alumnos.

Una vez decididos por la transformación del espacio docente, la realidad de construir aulas de este tipo plantea de antemano algunos interrogantes: ¿hace falta mucho espacio?, ¿son las condiciones de iluminación y sonido las adecuadas?, ¿servirá el aula como aula tradicional?, ¿qué se podrá hacer en ella?, etc. Así, se han abordado distintos estudios particulares para conseguir las mejores condiciones posibles, teniendo también presente el aspecto económico. El libro siempre ha sido un instrumento general para todos los estudiantes con las mismas características, para cambiar la organización y para individualizar el aprendizaje se utilizan los recursos que proporcionan las TIC. En nuestra concepción actual del aula, entendemos que el alumno puede disponer de la tradicional pizarra y del libro, de la integración de las TIC y también: de ordenador, campus virtual, Internet, videoconferencias etc. sin necesidad de desplazarse a otras áreas del recinto universitario.

Para la implementación de los cambios en la didáctica que hemos propuesto, resulta esencial disponer de un aula con el diseño y el equipamiento adecuados en la que se pueda llevar a cabo simultáneamente el programa teórico-práctico de las asignaturas Patología I y II, y de la Patología Especial, que facilite la integración de la práctica tradicional con microscopios y laminillas, el acceso simultaneo a casos vía Internet y a la página Web de la Unidad Didáctica, con la clase teórica y los nuevos materiales aportados por el profesor.

En nuestra propuesta respetamos la configuración tradicional del laboratorio de microscopía utilizado hasta ahora para las prácticas, por dos motivos esenciales: evita un cambio brusco en la intervención docente del profesorado, y permite albergar un número suficientemente grande de alumnos, microscopios y ordenadores, con facilidad para distribuirlos en grupos de menor tamaño.

Una vez transformada el aula y asumido este cambio por los profesores, apoyado por la institución y fomentado por los estudiantes, aspiramos a un crecimiento exponencial en el tiempo, es decir, que en los sucesivos años, los profesores con los diferentes estudiantes, enriquezcan permanentemente el sitio web de Patología, y de la misma manera, el aula sea un ejemplo de innovación en constante cambio con las nuevas tecnologías que seguirán, no nos cabe duda, impactando nuestro futuro docente.

Actualmente, nuestra aula busca ofrecer una solución técnica, racional, operativa y de bajo costo, aprovechando el espacio físico del que disponíamos con algunas modificaciones necesarias. Permite compartir experiencias de aprendizaje presencial, tanto individual como colaborativo, en un entorno virtual, que admite el acercamiento a las TIC al mismo tiempo que se desarrollan competencias y el uso crítico de las mismas. Con la transformación del aula pretendemos incorporar de manera sistemática y planificada, herramientas de aprendizaje a nuestros estudiantes.

Tanto alumnos como el profesor pueden acceder de manera simultánea a algunos de los contenidos de la asignatura de manera "on line" (a través de nuestra página web), con intercambio de ideas, discusiones presenciales y discusiones de casos en línea, a través de chats, foros, etc.

El aula de Patología es un moderno espacio didáctico equipado con aire acondicionado y una buena iluminación. Tiene una capacidad para 60 estudiantes. Cuenta con 20 mesas distribuidas en dos filas a cada lado del aula, con amplios pasillos entre ellas y dejando un gran espacio central en el que hay suficientes pupitres para que los estudiantes se agrupen alrededor del profesor cuando esto sea necesario. Las mesas son largas y anchas con tomas que permiten la instalación de Ethernet y de todos los elementos electrónicos de trabajo. Los espacios entre las mesas son amplios, permitiendo el acceso fácil de estudiantes a través de ellas.

Cada mesa tiene cupo para tres estudiantes cómodamente sentados, con tres microscopios y un monitor central. Cada estudiante puede poner sobre ellas sus libros, apuntes y laptops. De esta manera es posible el desarrollo de una clase teórica y práctica simultánea tanto en tiempo como en espacio. Los alumnos escriben normalmente en las mesas y toman el teclado y el ratón para trabajar de manera individual o en grupo cuando la actividad lo requiere.

En el fondo del aula hay un armario con múltiples puertas y divisiones. Dentro del mismo se guarda todo el material gastable, aparatos electrónicos y diferentes colecciones de laminillas, entre otros. La meseta del armario es amplia, y ancha y da soporte a los diferentes frascos de cristal con tapa, especialmente diseñados para la colección de las diversas piezas macroscópicas. La cabecera del aula cuenta con una gran pizarra, suficientes tizas blancas y de colores, elementos tradicionales y aún imprescindibles. Una pantalla central y un data-show colgado del techo, de fácil acceso y manejo. Un microscopio adaptado a un panel de control, permite al profesor dirigir la clase y proyectar tanto las imágenes del microscopio como las diapositivas por separado o simultáneamente durante la fase presencial y poder compartir con los estudiantes todas las herramientas de que dispone.

El profesor planifica cada clase presencial y, en función de esa planificación realiza, un cronograma que es facilitado en cada periodo académico a los estudiantes con suficiente tiempo de antelación, a través de las guías didácticas presentes en la página web de la asignatura. Esto permite que durante el desarrollo de la clase teórico-práctica existan 
posibilidades de interactividad y que aumente el grado de motivación y disposición del estudiante. Los contenidos temáticos son abordados por el profesor con la ayuda de las nuevas tecnologías, rememorando contenidos anteriores, realizando preguntas de control, facilitando los objetivos de esa clase concreta, y presentando los casos prácticos concretos. En determinado momento, ya previamente estipulado, los estudiantes tienen acceso a los casos prácticos. Estos casos siempre son propios del tema en estudio, o al menos guardan alguna conexión con él o con aspectos ya tratados.

El objetivo que se persigue con la observación directa de especímenes y con la revisión de imágenes o el estudio de casos problema basados en la Web, es poner ante el estudiante la realidad de las lesiones correspondientes a los temas que se van desarrollando. El profesor les ayuda a razonar sobre las lesiones, para obtener de la morfo-estructura su significado. El objetivo no es desarrollar una memoria fotográfica del aspecto de una lesión ni aprender a diagnosticarla; se trata de entender mejor la lesión de la que se está hablando, con su observación directa, trabajo que realiza de manera individual y personal.

Los estudiantes tienen acceso a la colección de piezas macroscópicas. Durante las horas de clase, pueden analizar, observar y describir estas piezas quirúrgicas. Su observación permite al estudiante desarrollar un pensamiento crítico y relacionar la lesión observada con los conocimientos adquiridos hasta ese momento. Posteriormente, hay una puesta en común donde se aclaran dudas y se somete a discusión los casos más difíciles. Todas las clases están permanentemente en la red (en la página de la asignatura), por lo que el estudiante puede acceder a ellas a través de sus propias laptops cada vez que necesite archivos de imágenes, esquemas y texto.

La concepción de este abordaje teórico-práctico simultáneo, apoyándonos en las nuevas herramientas que actualmente disponemos, tiene el propósito de vincular de manera atractiva y funcional la enseñanza de estos contenidos, motivando al estudiante a introducirse en el mundo de la Patología desde una nueva visión mucho más atractiva. Esta nueva perspectiva permite nuevas técnicas de dinámica grupal, la correlación clínico patológica y la comunicación bidireccional.

Entre algunas de las ventajas destacamos: todos los estudiantes y los profesores pueden participar en experiencias de trabajo colaborativo y de seminarios, con dispositivos didácticos virtuales. Simultáneamente los estudiantes pueden acceder a través de sus monitores, y de sus ordenadores portátiles, a las imágenes que el profesor emplea durante el desarrollo de la clase, e incluso a casos vía Internet, en el momento que lo precisen y de manera individualizada.

\section{Conclusión}

1. La Patología, en el Currículo de la Carrera de Medicina, toma gran relevancia debido a su importancia en la transferencia del conocimiento desde las ciencias básicas a las clínicas.

2. Nuevas estrategias para la enseñanza y el aprendizaje de la Patología: 2.2 El sistema b-learning permite acceso pleno a todos los recursos, tanto en la fase presencial como en la no presencial.
Además, permite el acceso fácil a las vías de comunicación estudiante-profesor haciendo uso de sus redes sociales página web y plataforma.

2.3 Durante la fase presencial, el abordaje simultáneo teóricopráctico, utilizando todas las herramientas de que disponemos (TIC), facilita la transferencia del conocimiento teórico a los problemas prácticos, logrando de esta manera un aprendizaje significativo.

\section{Referencias bibliográficas}

Ausubel D. (1980). Psicología educativa: un punto de vista cognoscitivo. México: Trillas.

Bixio, C. (2005). Enseñar a aprender. Construir un espacio colectivo de enseñanza-aprendizaje. Rosario: Homo sapiens.

Bloodgood, R. \& Ogilvie, R. (2006).Trends in histology laboratory teaching in United States medical schools, Anatomical Record (Part B: New Anat.), 289B, 169-175.

Boyd W. (1961). A textbook of Pathology. (7ma. ed). Philadelphia: Lea \& Febiger.

Contreras F. (1996). Memoria y proyecto docente. Facultad de Medicina, Universidad Autónoma de Madrid.

duBoulay C. (1997). Learning Pathology: Why?, How?, When?. Journal of Clinical Pathology, 50, 623-624.

Mayer R. (2009). Multimedia Learning (2ª ed). Cambridge: Cambridge University Press.

Reparaz, C., Sobrino, A. \& Mir, J. (2000): Integración Curricular de las Nuevas Tecnologías. Barcelona: Ariel.

Rogers A. (1993). Teaching adults. Oxford: Oxford University Press.

Rosai J. (1997). Guiding the surgeon's hand. Whashington: Armed Forces Institute of Pathology (AFIP).

Ruiz J., Mintzer M. \& Leipzig R. (2006). The Impact of E-Learning in Medical Education. Academic Medicine, 81, 207-212.

Sarsa, J. (2008). "Lo virtual" en las aulas presenciales: diseño de nuevos espacios educativos. Tesis no publicada. Universidad de Zaragoza.

Sharpe R., Benfield G., Roberts G. \& Francis R. (2006). The undergraduate experience of blended elearning: a review of UK literature and practice. Higher Education Academy, Extraído el 1 de junio de 2011 de http:// www.heacademy.ac.uk/projects/detail/Ir_2006_sharpe 